Journal of Geophysical Research: Atmospheres

\section{RESEARCH ARTICLE \\ 10.1002/2017JD028126 \\ High Spectral Resolution Spectroscopy of Sprites: A Natural Probe of the Mesosphere}

Key Points:

- High spectral resolution (0.24 nm)

spectra of sprites are presented

- High-resolution spectroscopy has

allowed us to resolve for the first time the internal (vibrorotational) structure of sprite FPS $\mathrm{N}_{2}$ emissions

- The temperatures of the explored region of the mesosphere (between 65 and $76 \mathrm{~km}$ ) range between $149 \mathrm{~K}$ and $226 \mathrm{~K}$; no measurable heating is found

Supporting Information: - Supporting Information S1

Correspondence to: F. J. Gordillo-Vázquez, vazquez@iaa.es

Citation:

Gordillo-Vázquez, F. J., Passas, M., Luque, A., Sánchez, J., van der Velde, O. A., \& Montanyà, J. (2018). High spectral resolution spectroscopy of sprites: A natural probe of the mesosphere. Journal of Geophysical Research: Atmospheres, 123, 2336-2346. https://doi.org/10.1002/2017JD028126

Received 29 NOV 2017 Accepted 29 JAN 2018 Accepted article online 6 FEB 2018 Published online 23 FEB 2018

(2)2018. American Geophysical Union. All Rights Reserved.

\author{
F. J. Gordillo-Vázquez ${ }^{1}$, M. Passas ${ }^{1}$, A. Luque ${ }^{1}$ (D) , J. Sánchez ${ }^{1}$, O. A. van der Velde ${ }^{2}$, \\ and J. Montanyá2 \\ ${ }^{1}$ Instituto de Astrofísica de Andalucía (IAA - CSIC), Granada, Spain, ${ }^{2}$ Department of Electrical Engineering, Universitat \\ Politécnica de Calatunya, Barcelona, Spain
}

\begin{abstract}
We present the first high spectral resolution $(0.24 \mathrm{~nm})$ spectra of sprites. These spectra were recorded in Europe in the summers and falls of 2015 and 2016 and during January 2017. The use of high spectral resolution has allowed us to resolve for the first time the internal (vibrorotational) structure of the sprite molecular $\mathrm{N}_{2}$ first positive system and to quantify the local gas (rotational) temperature of the mesosphere under the influence of sprites revealing that there is no measurable heating of the atmosphere associated to sprites at the altitudes explored. The temperatures of the explored region of the mesosphere range between $149 \mathrm{~K} \pm 10 \mathrm{~K}$ and $226 \mathrm{~K} \pm 20 \mathrm{~K}$. The recorded spectroscopic data also provide valuable quantitative information on the concentrations of some of the vibrational levels of molecular nitrogen involved in transient red-near-infrared optical emissions from sprites. The regions of the transient luminous events recorded with our spectrograph (equipped with a horizontally oriented slit) correspond to altitudes in the $66 \mathrm{~km}$ to $74 \mathrm{~km} \pm 5 \mathrm{~km}$ range.
\end{abstract}

\section{Introduction}

Soon after the publication of the first transient luminous event (TLE) images (of a sprite) recorded during the night of 22 to 23 September 1989 (Franz et al., 1990), the interest of the international community in TLE spectroscopic features started since spectra of TLE can inform about the type of molecules that produce optical emission, their degree of vibrational and electronic excitation, and if TLEs are able (or not) to produce a possible and measurable heating of the surrounding atmosphere. Previous works (Hampton et al., 1996; Mende et al., 1995) reported the first spectroscopic studies of the $\mathrm{N}_{2}$ first positive band system $\left(\mathrm{FPS}, \mathrm{B}^{3} \Pi_{g} \rightarrow \mathrm{A}^{3} \Sigma_{u}^{+}\right.$) from the "body" of sprites occurring between $70 \mathrm{~km}$ and $90 \mathrm{~km}$ of altitude. These early spectral investigations of TLEs used regular (30 fps) video recording in the visible and near-infrared spectral range ( $550 \mathrm{~nm}$ to $840 \mathrm{~nm}$ ) and low (above $6 \mathrm{~nm}$ ) spectral resolutions. Unfortunately, the number of spectroscopic campaigns of TLEs since the mid-1990s has been scarce. Later results (Bucsela et al., 2003; Morrill et al., 1998) reported spectroscopic investigations of the low-altitude "tendrils" (blurred motion of rapidly moving point-like structures (Liu \& Pasko, 2005; Stenbaek-Nielsen et al., 2007)) of sprites at $53 \mathrm{~km}$ and $57 \mathrm{~km}$ covering from $600 \mathrm{~nm}$ up to $900 \mathrm{~nm}$ with low spectral resolutions of $7 \mathrm{~nm}$ and $11 \mathrm{~nm}$, respectively. More recently, altitude-resolved sprite spectra of the $\mathrm{N}_{2}$ FPS have been reported with an imaging (vertical) slit spectrograph within $640 \mathrm{~nm}$ to $820 \mathrm{~nm}$ with $3 \mathrm{~ms}$ (300 fps) temporal and $3 \mathrm{~nm}$ spectral resolutions (Kanmae et al., 2007).

Regarding near-ultraviolet (below $400 \mathrm{~nm}$ ) spectra of sprites, the only measurements available to date (without correction for instrument response function) are those focusing on optical emissions of the $\mathrm{N}_{2}$ second positive band system (SPS, $\mathrm{C}^{3} \Pi_{u} \rightarrow \mathrm{B}^{3} \Pi_{g}$ ) from the "body" of sprites at $65 \mathrm{~km}$ altitude (Heavner et al., 2010). This near-ultraviolet sprite spectrum was recorded during the EXL98 observation campaign from an aircraft at $14 \mathrm{~km}$ altitude and using $\sim 4 \mathrm{~nm}$ spectral resolution and $60 \mathrm{fps}$ recording rate.

High-speed (10,000 fps) slitless spectroscopy of sprite streamer heads and column-shaped glows were reported for the first time in 2010 from aircraft observations including blue spectral features (Kanmae et al., 2010a) and from ground-based facilities (Kanmae et al., 2010b) with the spectra consisting only of the $\mathrm{N}_{2}$ FPS due to the atmospheric absorption of the blue component through the long line of sight.

The different types of TLEs occurring in the Earth's atmosphere from the lower stratosphere to the upper mesosphere can be considered as natural probes to learn about the properties of the middle and upper atmosphere 
of our planet through remote determination of key properties such as the gas temperature or the degree of excitation and ionization of atoms and molecules caused by TLEs in the atmosphere. In this regard, systematic spectroscopic campaigns of optical emissions from all sorts of TLEs can allow us to remotely determine important atmospheric properties that would be difficult to obtain in other ways. Unfortunately, the spectrographs used in the dispersed TLE spectroscopic campaigns carried out so far were originally designed for aurora spectroscopy (Hallinan et al., 1985) and the best spectral resolution achieved to date is $\sim 3 \mathrm{~nm}$ which it is not enough to spectrally resolve the different low-lying vibrational transitions of the $\mathrm{N}_{2}$ FPS.

The lack of TLE spectroscopic observations with high spectral resolution motivated us to build a TLE-dedicated instrument called GRAnada Sprite Spectrograph and Polarimeter (GRASSP). This spectrograph has a mean spectral resolution of $0.24 \mathrm{~nm}$ (with a linear dispersion of $0.07 \mathrm{~nm} /$ pixel) and covers the wavelength range between $700 \mathrm{~nm}$ and $800 \mathrm{~nm}$ with $40 \mathrm{~ms}$ (25 fps) temporal resolution (Passas, Sánchez, et al., 2016) (see supporting information for details). GRASSP is in operation for ground-based systematic imaging and spectroscopic surveys of all sorts of TLEs in Europe since October 2012 when the first GRASSP version (Parra-Rojas, Passas, et al., 2013) was deployed in the Calar Alto Astronomical Observatory (CAHA) (3713'23"N $\left.232^{\prime} 45^{\prime \prime} \mathrm{W}\right)$ placed 2,168 m above sea level in southeast Spain. GRASSP recorded from Calar Alto Astronomical Observatory many images of distant TLEs (Passas et al., 2014) and carried out meteor spectroscopy (Passas, Madiedo, \& Gordillo-Vazquez , 2016). An upgraded version of GRASSP is now available and relocated (since May 2015) in Castellgali (Barcelona, 4140'36"N, 150'24"E) placed $266 \mathrm{~m}$ above sea level and close to the Ebro valley.

As we describe below, GRASSP has allowed to resolve for the first time the vibrational bands of $\mathrm{N}_{2}$ FPS in transient air plasma spectra generated by TLEs and to remotely determine the gas temperature in the mesosphere and if there is any measurable heating due to sprites at the altitudes explored. We have calculated the approximate altitudes of the TLE regions whose spectra are recorded, and we have correlated them with the derived gas temperatures. A more detailed discussion on the methodology followed for sprite altitude determination can be found in the supporting information (São Sabbas et al., 2003; van der Velde \& Montanyà, 2016). Furthermore, we present quantitative measurements of the relative vibrational concentrations of the emitting electronic state $\mathrm{N}_{2}\left(\mathrm{~B}^{3} \Pi_{g}, v\right)$ from carrot, single-column, and multicolumn-like sprites and compare them with previous measurements and theoretical calculations.

\section{Observations and Results}

TLE activity during the 2015 campaign was relatively high in southwestern Europe. However, the number of sprites recorded with GRASSP was low. A total of three events were recorded with the spectrograph with good quality data obtained from carrot and column-like sprites occurring in a range between $350 \mathrm{~km}$ and about $800 \mathrm{~km}$. In general, spectra are only present in one frame due to the low available temporal resolution and the peaked brightness profile of sprites.

Sprite spectra (see Table 1) were recorded from Castellgali, Spain, $266 \mathrm{~m}$ above sea level at 01:15:26 UTC (event 1) and 01:20:55 UTC (event 2) on 24 August 2015 and at 04:07:44 UTC on 28 September 2015 (events $3 a$ and 3b). Two distant clusters of carrot-like sprites were recorded on 24 August 2015 over a thunderstorm in northern Italy (see Figure 1, top). On 28 September 2015 a close column-like sprite was recorded over a thunderstorm in the south of the Balearic islands (see Figure 1, bottom). The maps in Figure 1 show the location of the parent lightning-producing sprites detected at 01:15:26 UTC and 01:20:55 UTC on 24 August 2015 (top panel) and at 04:07:44 UTC on 28 September 2015 (bottom panel). The lightning detection network used was Llghtning detection NETwork (LINET) (Betz et al., 2008). The images shown in Figure 1 were recorded by Meteosat Second Generation, and the color bar in the lower right corner of the top image refers to cloud top temperatures. Regarding the size and color of the circles in Figure 1, the size scales with peak current (which is labeled). The different colors of the circles refer to the number of sprite events produced by the lightning events of which we got spectra.

Figure 2 shows scene camera images of two carrot sprites (events 1 and 2) and one multicolumn sprite (events $3 \mathrm{a}$ and $3 \mathrm{~b}$ ).

The TLE spectroscopic campaigns during 2016 and January 2017 were more fruitful than the one of 2015. Up to the end of January 2017 a total of 32 events were recorded with GRASSP. The events included carrot and column-like sprites as well as precursory halos to some of the detected sprites. Figure 2 (third and fourth rows) corresponds to the 2016 and 2017 sprites analyzed in this work. 
Table 1

Altitudes and Rotational (Gas) Temperatures Derived From High-Resolution Spectra of Sprite Events With Different Morphology Captured Through Our 2015, 2016, and January 2017 TLE Spectroscopic Campaigns in Spain

\begin{tabular}{lcccccc}
\hline Event & ${\text { Altitude }(\mathrm{km})^{\mathrm{a}}}^{\mathrm{a}}$ & $T_{\text {rot }}(\mathrm{K})$ & Sprite type & Time (UTC) and date & $T_{L}(\mathrm{~K})$ & $T_{S}(\mathrm{~K})$ \\
\hline 1 & $71-73$ & $185 \pm 10$ & Carrot & 01:15:26 on 24 August 2015 & $204 \pm 2$ & $201 \pm 2$ \\
2 & $70-72$ & $190 \pm 11$ & Carrot & 01:20:55 on 24 August 2015 & $204 \pm 2$ & $201 \pm 2$ \\
3a & $66-67$ & $149 \pm 10$ & Single column & 04:07:44 on 28 September 2015 & $223 \pm 2$ & $221 \pm 2$ \\
3b & $66-67$ & $210 \pm 24$ & Single column & 04:07:44 on 28 September 2015 & $223 \pm 2$ & $221 \pm 2$ \\
4 & $72-74$ & $178 \pm 10$ & Column carrot & 01:12:59 on 15 September 2016 & $209 \pm 2$ & $202 \pm 2$ \\
5 & $70-71$ & $164 \pm 18$ & Multicolumn & 02:23:07 on 16 November 2016 & $218 \pm 2$ & $211 \pm 2$ \\
6 & $71-72$ & $226 \pm 17$ & Multicolumn & 23:37:32 on 17 January 2017 & $224 \pm 2$ & $215 \pm 2$ \\
7 & $69-70$ & $201 \pm 20$ & Multicolumn & 23:40:35 on 17 January 2017 & $224 \pm 2$ & $215 \pm 2$ \\
\hline
\end{tabular}

Note. Also shown (two columns to the right) are $T_{L}$ and $T_{S}$. $T_{L}$ stands for the mean (for the fifteenth of each month) gas temperatures as derived from two Rayleigh lidars operated between 1978 and 1989 in the south of France $\left(44^{\circ} \mathrm{N}, 6^{\circ} \mathrm{E}\right.$ and $44^{\circ} \mathrm{N}, 1^{\circ} \mathrm{W}$ ) (Hauchecorne et al., 1991). $T_{S}$ stands for the gas temperature measured by the instrument SABER on board the TIMED satellite in a region centered in the location of each of the sprite corresponding parent lightning $\left( \pm 5^{\circ}\right.$ latitude and $\pm 10^{\circ}$ longitude) within an altitude range between $66 \mathrm{~km}$ and $74 \mathrm{~km} \pm 5 \mathrm{~km}$ and in a time interval $\pm 4 \mathrm{~h}$ with respect

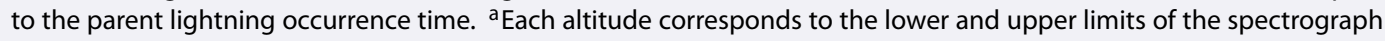
slit with an uncertainty of $\pm 5 \mathrm{~km}$.

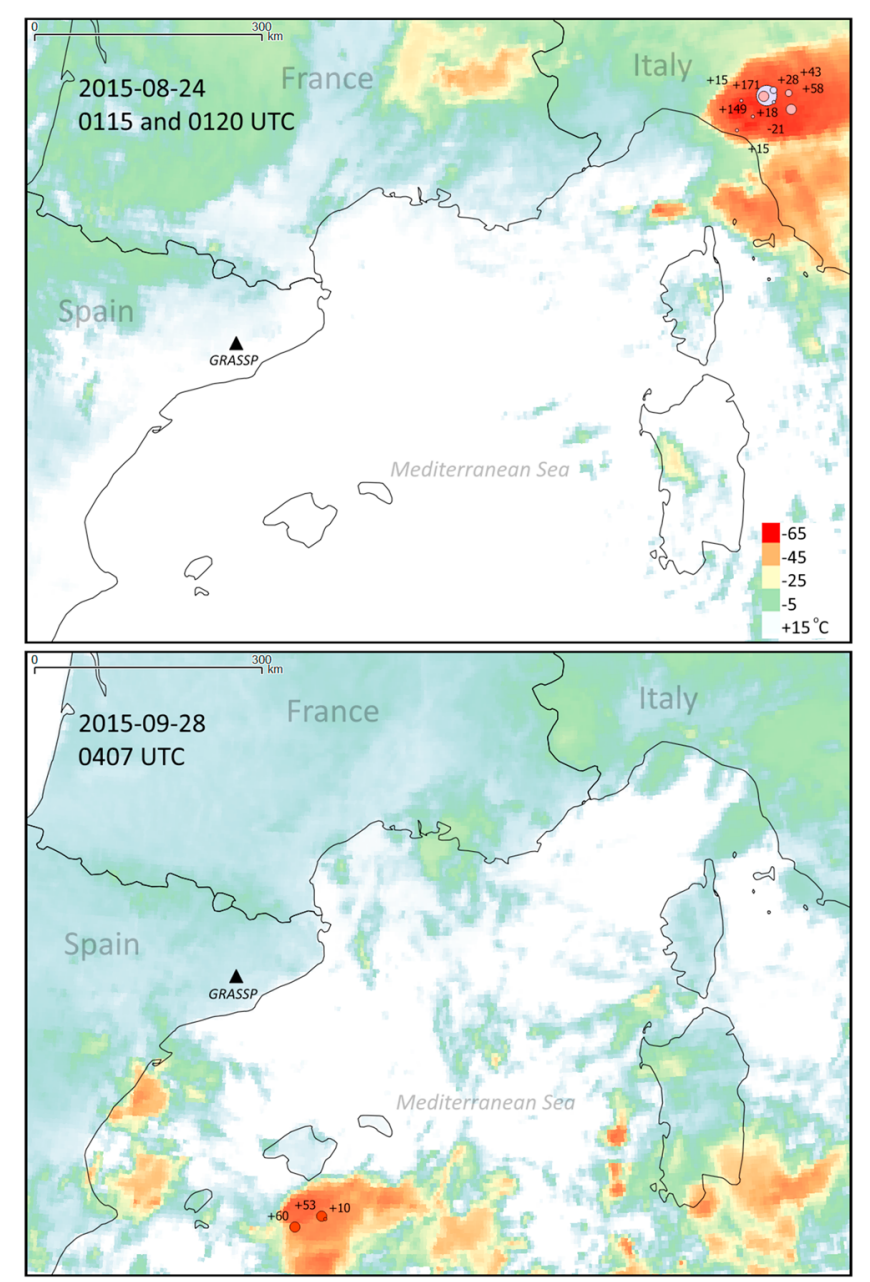

Figure 1. Location (provided by LINET) of the parent lightning-producing carrot-like sprites detected at 01:15 UTC and 01:20 UTC on 24 August 2015 (top) and column-like sprites detected at 04:07 UTC on 28 September 2015 (bottom). The location of GRASSP in Castellgali (black triangle) and the thunderstorm systems (reddish shadows) are also shown. The circles and numbers indicate the parent lightning position, and corresponding peak intensities (in kA) and polarities $( \pm)$, respectively 

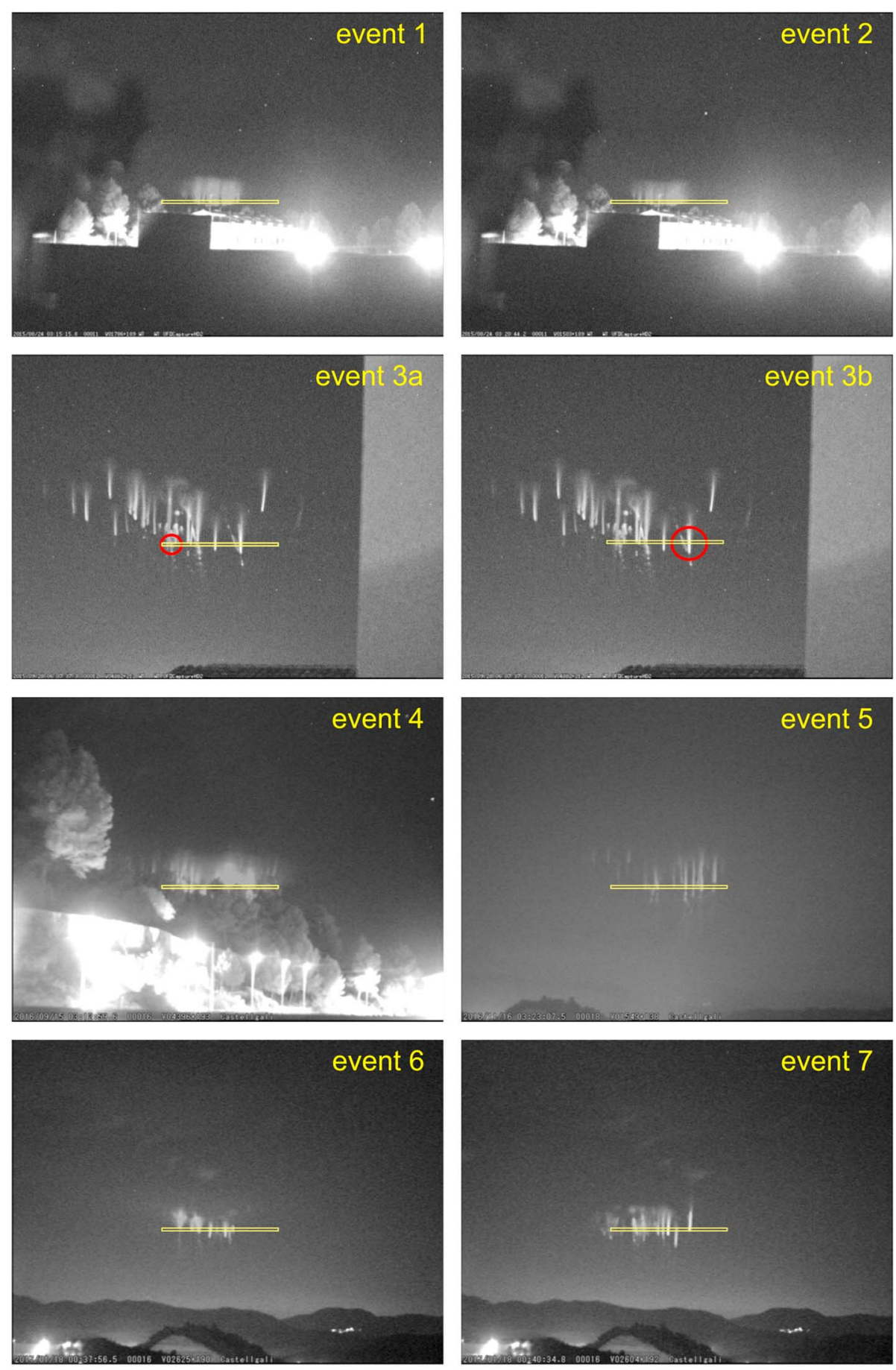

Figure 2. Scene camera images with the spectrograph slit superimposed showing sprites corresponding to, respectively, event 1 (first row, left), event 2 (first row, right), events 3a and 3b (second row, left and right), event 4 (third row, left), event 5 (third row, right), event 6 (fourth row, left), and event 7 (fourth row, right) as shown in Table 1. The red circles in the images of the second row indicate the sprite column for which the spectra are shown in Figures 3 and 4.

The 2016 and January 2017 TLE spectra were also recorded from Castellgali, Spain (see scene camera images in Figure 2, third and fourth rows). The TLE spectra on 14-15 September 2016 were recorded over southeastern France (area around $4-8^{\circ} \mathrm{E}$ and $43-46^{\circ} \mathrm{N}$ ) at 23:47:20 UTC, 23:55:35 UTC, 01:12:59 UTC (event 4), and 01:16:38 UTC. Two additional carrot sprite spectra were recorded on 21 October 2016 over east of Menorca Island (Spain) at 01:24:19 UTC and 01:37:01 UTC. A total of 24 sprite spectra were recorded between November 2016 and January 2017. However, only some of them presented good signal-to-noise ratio (SNR). 

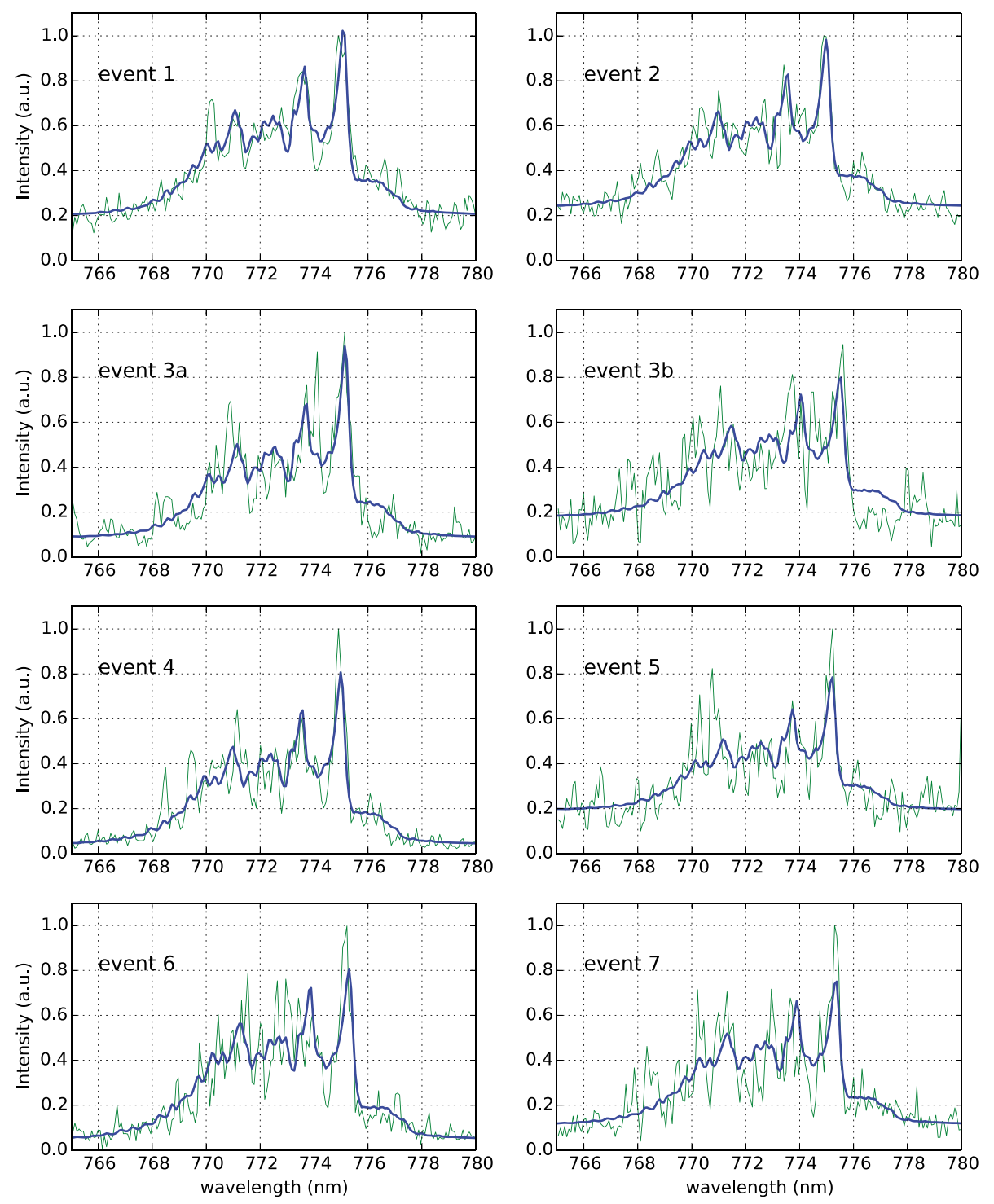

Figure 3. Spectral fitting (blue line) of recorded (green line) carrot and multicolumn sprite spectra of which images are shown in Figure 2. The spectra correspond to the $(2,0)$ rovibronic transition of the $\mathrm{N}_{2}\left(B^{3} \Pi_{g} \rightarrow A^{3} \Sigma_{u}^{+}\right)$system in the recorded and synthetic spectra. The observed spectra were recorded with a mean spectral resolution of $0.24 \mathrm{~nm}$, while the synthetic spectra were calculated using a fixed resolution of $0.24 \mathrm{~nm}$.

In particular, we have chosen the ones that occurred at 02:23:07 UTC (event 5) on 16 November 2016 and at 23:37:32 (UTC) and 23:40:35 UTC (event 7) on 17 January 2017.

The $\mathrm{N}_{2}$ FPS $(2,0)$ rovibronic band spectra of the two recorded carrot-like sprites (event 1 and event 2) were fitted with synthetic spectra following previously described procedures (Biloiu et al., 2007; Herzberg, 1950; Parra-Rojas, Passas, et al., 2013). A more detailed discussion on the fitting procedure can be found in the supporting information (Simek, 1994; Simek \& De Benedictis, 1995). Figure 3 shows good agreement between the recorded (green line) and fitted synthetic (blue line) spectra of two carrot sprite spectra corresponding to event 1 and event 2. Due to the long slant path (between 300 and around $800 \mathrm{~km}$ ) the spectroscopic signal from TLEs can be strongly affected by absorption from various atmospheric constituents $\left(\mathrm{O}_{2}, \mathrm{H}_{2} \mathrm{O}\right.$, and $\left.\mathrm{CO}_{2}\right)$. To quantify this effect, we have estimated atmospheric transmittance using MODTRAN 5 (Berk et al., 2005) in the 700-800 nm spectral range with the observing site (Castellgali, Spain) at only $266 \mathrm{~m}$ above sea level (see transmittance curves in supporting information). All spectra shown in Figure 3 clearly exhibit the expected three main peaks of the $(2,0)$ band of the $\mathrm{N}_{2}$ FPS located, respectively, in the spectral positions $774.8-775.4 \mathrm{~nm}$ (band head peak), 773.5-774.1, and 771.0-771.6 nm (see supporting information) (Simek, 1994). 

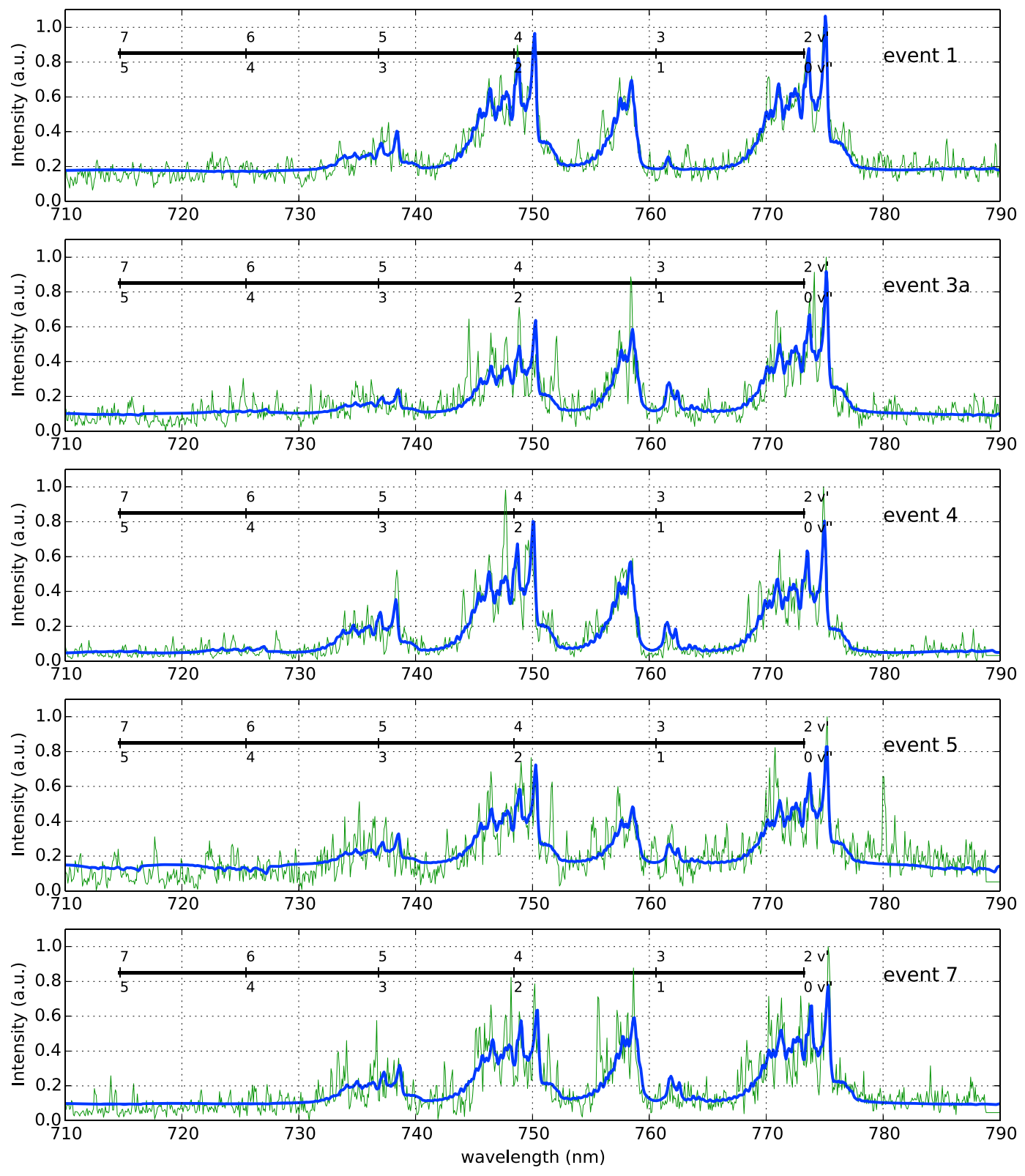

Figure 4. Spectral fitting (blue line) between $710 \mathrm{~nm}$ and $790 \mathrm{~nm}$ of the recorded (green line) spectra corresponding to event 1 , event $3 a$, event 4 , event 5 , and event 7 according to Table 1 . The synthetic (fitted) spectra are generated using a spectral resolution of $0.24 \mathrm{~nm}$. The different rovibronic bands $\left(v^{\prime}, v^{\prime \prime}\right)$ of the $\mathrm{N}_{2}\left(\mathrm{~B}^{3} \Pi_{g} \rightarrow \mathrm{A}^{3} \Sigma_{u}^{+}\right)$system are marked. The used $T_{\text {rot }}$ are extracted from previous rotational fitting of the $(2,0)$ band (see Figure 3 ). The original recorded spectra are normalized to the $(2,0)$ band head peak $(774.8 \mathrm{~nm}$ to $775.4 \mathrm{~nm})$.

The rotational gas temperatures $\left(T_{\text {rot }}\right)$ from the fitted spectra in Figure 3 corresponding to two different carrot sprite events (event 1 and event 2), captured during the 2015 campaign, are $185 \mathrm{~K} \pm 10 \mathrm{~K}$ (event 1 ) and $190 \mathrm{~K} \pm 11 \mathrm{~K}$ (event 2). The calculated uncertainties of the derived gas temperatures correspond to the standard deviation $\sigma$ (square root of the variance) obtained from the numerical least squares fitting of synthetic spectra to the recorded spectra. These estimated uncertainties are probably underestimating the real error.

Also shown in Figure 3 are the spectra of different regions of a multicolumn sprite recorded at 04:07:44 UTC on 28 September 2015. In particular, the recorded (green line) and fitted spectra (blue line) are those corresponding to event $3 \mathrm{a}$ and event $3 \mathrm{~b}$ which are marked with red circles in Figure 2. The recorded column sprite spectra (second row of Figure 3) are a bit noiser than the ones of the carrot sprites (Figure 3, first row) because the available optical signal is weaker. According to parent lightning location, the analyzed column-like sprites occurred 
closer $(\simeq 350 \mathrm{~km})$ to the observing site (see Figure 1, bottom) than the detected carrot sprites $(\simeq 800 \mathrm{~km})$. However, the spectral fit of the $(2,0)$ rovibronic transition in the recorded multicolumn spectra remains quite reasonable with clear visibility of the three main peaks of the $(2,0)$ band of the $\mathrm{N}_{2}$ FPS. The rotational gas temperatures $\left(T_{\text {rot }}\right)$ derived from the synthetic spectra fitting to single-column spectra shown in Figure 3 are $149 \mathrm{~K} \pm 10 \mathrm{~K}$ (event $3 \mathrm{a}$ ) and $210 \mathrm{~K} \pm 24 \mathrm{~K}$ (event $3 \mathrm{~b}$ ).

The spectrum of event 4 corresponds to the left-side columnar structure of the carrot sprite partially hidden by trees as seen in Figure 2. In all these spectra the three main peaks of the $(2,0)$ band of the $N_{2}$ FPS are distinctly visible.

The rotational gas temperatures $\left(T_{\text {rot }}\right)$ derived from the 2016 and 2017 fitted spectra in Figure 3 are $178 \mathrm{~K} \pm 10 \mathrm{~K}$ (event 4), $164 \mathrm{~K} \pm 18 \mathrm{~K}$ (event 5), $226 \mathrm{~K} \pm 17 \mathrm{~K}$ (event 6), and $201 \mathrm{~K} \pm 20 \mathrm{~K}$ (event 7). Table 1 shows that the derived gas temperature fluctuates depending on the type of sprite probing different altitudes of the mesosphere.

Figure 4 shows the full (green line) spectra recorded by GRASSP and the corresponding fitted spectra (blue line) using the same $0.24 \mathrm{~nm}$ spectral resolution. The gas temperatures used to generate the fitted synthetic spectra in Figure 4 are shown in Table 1 , and they are derived from the rotational fits of the $(2,0)$ band in the spectra of events $1,3 a, 4,5$, and 7 (see Figure 3). The synthetic spectra are for neutral $\mathrm{N}_{2}$ only, and it includes six $\Delta v=2$ rovibronic band transitions marked in the plots of the $\mathrm{N}_{2}\left(\mathrm{~B}^{3} \Pi_{g} \rightarrow \mathrm{A}^{3} \Sigma_{u}^{+}\right)$electronic transition.

\section{Discussion}

Sprite spectra recorded with GRASSP during the spectroscopic campaigns of 2015, 2016, and January 2017 from Spain are the first high-resolution spectra of TLEs to date. The spectral resolution used $(0.24 \mathrm{~nm})$ has allowed us to resolve the rovibrational structure of $\Delta v=2$ bands of the $\mathrm{N}_{2}$ FPS within the explored spectral range $(700-800 \mathrm{~nm})$ from an almost sea level $(266 \mathrm{~m})$ observation site. We captured high-resolution spectra of full carrot sprites, single-column sprites (see red circles in Figure 2, second row) and multicolumn sprites. Figure 2 shows the different morphologies of recorded sprites covering a range of altitudes between 66 and $67 \mathrm{~km} \pm 5 \mathrm{~km}$ (lower region of a multicolumn sprite-event 3) and 71-72 km $\pm 5 \mathrm{~km}$ (column spriteevent 6). Note that we give the altitudes (and corresponding error) associated to the lower and upper limits of the spectrograph slit.

Figure 3 shows high-resolution spectra of the $(2,0)$ band of the $N_{2}$ FPS associated to each of the sprites in Figure 2 . We can see that the $(2,0)$ band is well resolved and that the spectra with the best SNR correspond to carrot sprites since they produce higher and more uniform optical signal in the spectrograph slit than the narrow regions of the recorded single and multicolumn sprites.

Contrary to the assumed rotational (gas) temperatures reported in previous low-resolution sprite spectra (Hampton et al., 1996; Kanmae et al., 2007; Mende et al., 1995), our high-resolution TLE spectroscopic campaigns have allowed us to extract the rotational temperature from the fitting shown in Figure 3 of the recorded and synthetic spectra of the $\mathrm{N}_{2}$ FPS $(2,0)$ band.

The analysis of highly resolved spectra associated with the altitude localization of sprites makes them a useful "mesospheric thermometer." Table 1 shows that the derived gas temperature fluctuates depending on the type of sprite probing different altitudes of the mesosphere. The mesospheric temperatures obtained from high-resolution spectra of sprites are in reasonable agreement with previous temperature measurements performed from 1978 to 1989 by two Rayleigh lidars (Hauchecorne et al., 1991) in the south of France $\left(44^{\circ} \mathrm{N}, 6^{\circ} \mathrm{E}\right.$ and $\left.44^{\circ} \mathrm{N}, 1^{\circ} \mathrm{W}\right)$ close to our spectroscopic recordings. Table 1 also includes mesospheric temperatures recorded by the instrument Sounding of the Atmosphere using Broadband Emission Radiometry (SABER) on board the satellite TIMED (Thermosphere, lonosphere, Mesosphere Energetics and Dynamics) in the region, and altitudes and times explored in this work. SABER provides temperature profiles from 20 to $105 \mathrm{~km}$, retrieved from $\mathrm{CO}_{2} 15 \mu \mathrm{m}$ emission with $1.5-2 \mathrm{~K}$ errors in the $60-80 \mathrm{~km}$ range (García-Comas et al., 2008). Most of the temperature measurements are $20 \mathrm{~K}$ below available lidar and SABER measurements except in one case-event $3 \mathrm{a}$-where it is about $70 \mathrm{~K}$ below. This is only explained by the uncertainty of the fitting procedure. The quality of the fitting is controlled by the noise in the observed spectra. Our lowest error is $\simeq 5 \%(185 \mathrm{~K} \pm 10 \mathrm{~K})$, while the errors of both the lidars and SABER are $\simeq 1 \%$. Sprite spectra are transient by nature, and this introduces an additional complication. Spectroscopy of a single-column sprite is specially challenging because much less photons are collected (considered) and the SNR is worse, producing 
noisier spectra. This is the case of event $3 a$ where the difference between the lidar and SABER gas temperatures is almost $70 \mathrm{~K}$. There is also a lack of statistics; to probe the mesosphere temperature by means of fitting TLE spectra with theoretical models, we need a larger amount of data that might allow us to measure the mesosphere temperature in a more consistent way.

The temperature values obtained indicate that there is no measurable heating of the mesosphere due to the presence of sprites within the range of altitudes explored in this work. Due to the scarce number of measurements, it is hard to identify a clear trend in the temperatures shown in Table 1 (Lopez-Gonzalez et al., 2007). In this regard, one is tempted to see a seasonal behavior in the measured temperatures of the mesosphere, but available data are too few.

It is worth mentioning that the recorded high-resolution spectra only show spectroscopic features of neutral molecular nitrogen. In particular, the recorded high-resolution $(2,0)$ band spectra of the $\mathrm{N}_{2}$ FPS in carrot, single-column, and multicolumn sprites did not show any traces of the strong $777.4 \mathrm{~nm}$ atomic oxygen (O I) triplet line usually seen in lightning spectra in the troposphere. While the mechanisms of energy dissipation in lightning are controlled by high currents (of typically tens of kA and tens of milisecond duration) and very frequent collisions in a high-pressure environment, energy dissipation in sprites and halos is controlled by impulsive high reduced electric fields transferring energy to electrons in a low-pressure ambient. Therefore, energetic electrons in sprites and halos can excite molecules, but also, depending on how high the gained energy is, electrons can dissociate and ionize $\mathrm{N}_{2}$ and/or $\mathrm{O}_{2}$ molecules (Adachi et al., 2008; Gordillo-Vázquez et al., 2011; Hiraki, 2010; Luque \& Ebert, 2009; Parra-Rojas, Luque, \& Gordillo-Vazquez, 2013; Williams, 2012). Sprites generally exhibit an upper $(85-75 \mathrm{~km})$ diffuse structure and a lower $(75-40 \mathrm{~km})$ filamentary region where thousands of streamers develop and where reduced electric fields can reach values of 400-600 Td (Luque \& Ebert, 2010; Pasko et al., 1998; Stenbaek-Nielsen et al., 2010) that activate complex nonequilibrium electron-driven kinetics in low-pressure air (Gordillo-Vázquez, 2008; Parra-Rojas et al., 2015; Sentman et al., 2008). In particular, the production of the ${ }^{5} \mathrm{P}$ electronic state of $\mathrm{O} \mathrm{I}$, whose radiative decay produces the $777.4 \mathrm{~nm}$ line, could be activated by (i) electron impact dissociative excitation of $\mathrm{O}_{2}\left(\mathrm{e}+\mathrm{O}_{2} \rightarrow \mathrm{O}+\mathrm{O}\left({ }^{5} \mathrm{P}\right)+e\right)$ generating ground state $\mathrm{O} I$ atoms and electronically excited $\mathrm{O}\left({ }^{5} \mathrm{P}\right)$ atoms and (ii) direct electron impact excitation of ground state free ambient $\mathrm{O}$ I atoms producing electronically excited $\mathrm{O}\left({ }^{5} \mathrm{P}\right)$ atoms $\left(\mathrm{e}+\mathrm{O} \rightarrow \mathrm{O}\left({ }^{5} \mathrm{P}\right)+\mathrm{e}\right)$. The rate coefficients of mechanisms (i) and (ii) in low-pressure (mesospheric) dry air increase as the reduced electric field grows, but the production of $\mathrm{O}\left({ }^{5} \mathrm{P}\right)$ by dissociative excitation is always significantly higher than the one by direct electron impact excitation from ground state oxygen atoms. Therefore, it could be possible that $777.4 \mathrm{~nm}$ optical emissions appear in well-resolved sprite spectra. The possible $777.4 \mathrm{~nm}$ emission would not be uniformly produced through the entire body of the sprite but only in regions with high enough reduced electric fields like well-developed streamers in sprites and/or in regions of sprites where streamer reconnections occur. However, none of the spectra of our detected sprites in the $66 \mathrm{~km}$ to $74 \mathrm{~km} \pm 5 \mathrm{~km}$ region exhibit the characteristic $777.4 \mathrm{~nm}$ lightning emission feature that could be more probable in lower-altitude $(50-55 \mathrm{~km})$ regions of sprites.

Figure 4 shows high-resolution observed spectra (green line) of carrot sprites (first and third panels), single-column sprite (second panel), and multicolumn sprites (fourth and fifth panels). None of the spectra shown in Figure 4 indicates the possible presence of optical transitions associated to the $(2,0)$ band (around $785 \mathrm{~nm}$ ) of $\mathrm{N}_{2}^{+}$Meinel emissions or to the $777.4 \mathrm{~nm}$ emission from the ${ }^{5} \mathrm{P}$ electronic state of $\mathrm{O}$ I. The fit of high-resolution $(0.24 \mathrm{~nm})$ synthetic spectra (blue line) to observed sprite spectra shown in Figure 4 is very good for carrot sprites, while it is reasonable for the others. These fits allow us to derive the vibrational distribution function (VDF) or populations of the lower (from $v^{\prime}=2$ up to $v^{\prime}=7$ ) vibrational levels of the $B^{3} \Pi_{g}$ electronic state of $\mathrm{N}_{2}$ that are shown in Figure 5 for carrot (left) and single-column and multicolumn (right) sprites, respectively. The uncertainties in the derived relative vibrational populations range between $2 \%$ and $5 \%$. The VDFs derived from fits are compared to (1) VDF of the $B^{3} \Pi_{g}$ state from previous spectroscopic observations (squares and dashed green lines) (Kanmae et al., 2007) of carrot-like sprites at $71-75 \mathrm{~km}$ altitude using $3 \mathrm{~nm}$ spectral resolution and (2) model-calculated sprite VDFs (open circles and dashed blue line) at $74 \mathrm{~km}$ altitude (Gordillo-Vázquez et al., 2012; Luque \& Gordillo-Vázquez, 2011). In particular, Figure 5 (left) includes VDFs of carrot sprites corresponding to events 1 and 4 . Our carrot sprite VDFs deviate from previous observations for $v^{\prime}=3$ and $v^{\prime}=4$, while the agreement with VDF model predictions is reasonable except for $v^{\prime}=4$. Note that our two shown VDFs from carrot sprites observed at similar altitudes exhibit values close to those of Kanmae et al. (2007). Figure 5 (right) includes VDFs from events 3a, 3b, 5, and 7. The altitude dependence of event 5 and event 7 VDFs follows the trend previously reported within a carrot sprite (Kanmae et al., 2007), 

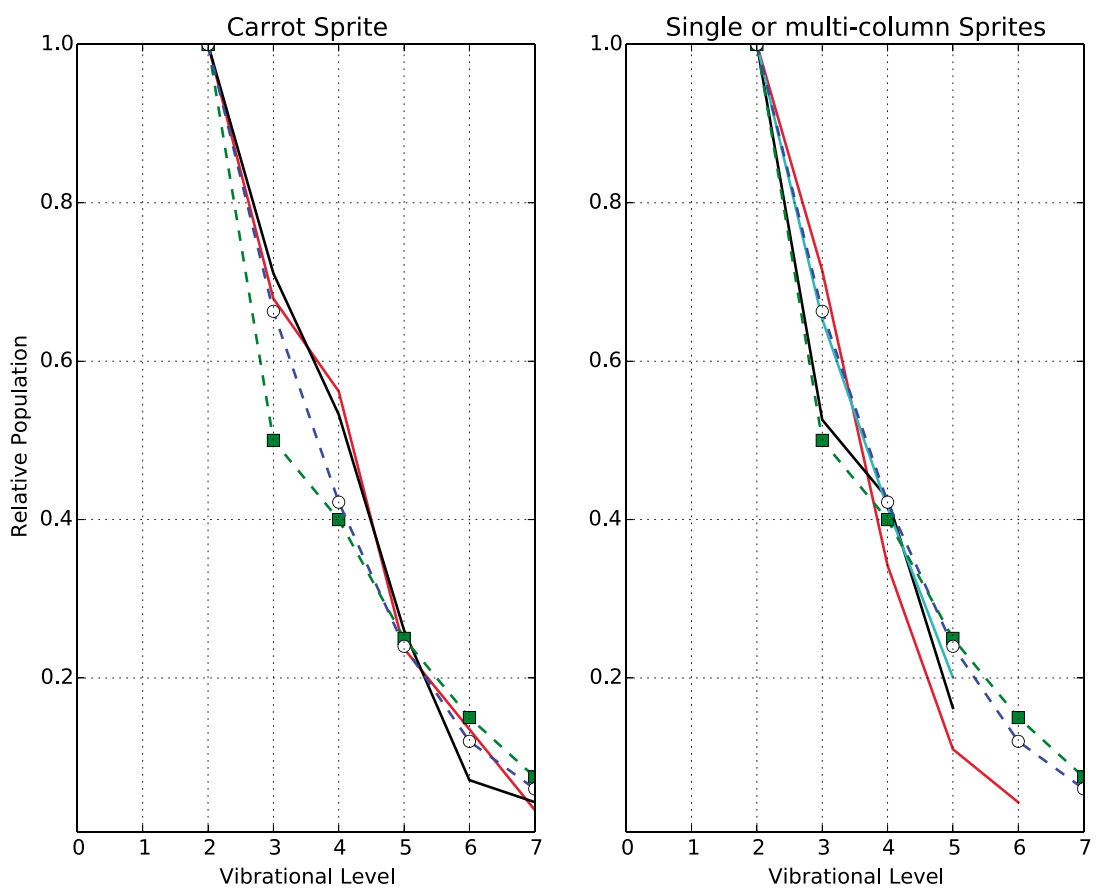

Figure 5. Relative populations of the vibrational levels (or vibrational distribution function (VDF)) of the $\mathrm{N}_{2}\left(\mathrm{~B}^{3} \Pi_{g}, v^{\prime}\right)$ electronic state as obtained from recorded spectra of carrot-like (left panel, events 1 (red line) and 4 (black line)) and column-like sprites (right panel, events 3a (red line), 5 (black line), and 7 (cyan line)). Also shown are (1) altitude-resolved VDFs derived from previously reported spectroscopic observations (squares and dashed green lines) of carrot-like sprites recorded at 71-75 km altitude using $3 \mathrm{~nm}$ spectral resolution (Kanmae et al., 2007) and (2) calculated sprite VDFs at 74 km altitude (Gordillo-Vázquez et al., 2012; Luque \& Gordillo-Vázquez, 2011) (open circles and dashed blue line).

Acknowledgments

This work was supported by the Spanish Ministry of Science and Innovation, MINECO, under projects FIS2014-61774-EXP, ESP2015-69909-C5-2-R, and ESP2017-86263-C4-4-R, and by the EU through the FEDER program. The contribution of O. V. and J. M. was supported by projects ESP2013-48032-C5-3-R and ESP2015-69909-C5-5-R. A. L. was supported by the European Research Council (ERC) under the European Union H2020 program/ERC grant agreement 681257. We acknowledge NowCast for providing LINET lightning data for research purposes. We also acknowledge AEMET (Spanish Meteorological Service) for providing some lightning data corresponding to January 2017. Finally, we thank Maya Garcia-Comas and the SABER team for making temperature data publicly available through https://saber.gats-inc.com. The spectroscopic data presented here are available from figshare repository at https://figshare.com/s/

ac4f082631b7090eba02. Alternatively, the same spectroscopic data available through figshare are also available after a request is made to authors F. J. G. V. (vazquez@iaa.es), M. P. (passasv@iaa.es), or A. L. (aluque@iaa.es). that is, higher vibrational population for sprites at higher altitudes. However, the VDF of the single-column sprite (events $3 a$ and $3 b$ ) does not follow the above mentioned altitude-dependent trend for $v^{\prime}=3$.

\section{Summary}

High-resolution systematic spectroscopic campaigns of TLEs have been carried out in Europe during 2015, 2016 , and early 2017. It is the first time that the $(2,0),(3,1),(4,2)$, and $(5,3)$ rovibrational bands of the $N_{2}$ FPS are well resolved in sprite spectra. We found that the rotational (gas) temperature derived from sprite spectra changes between $149 \mathrm{~K} \pm 10 \mathrm{~K}$ at $66-67 \mathrm{~km} \pm 5 \mathrm{~km}$ in September 2015 and $226 \mathrm{~K} \pm 17 \mathrm{~K}$ at $71-72 \mathrm{~km} \pm 5 \mathrm{~km}$ in January 2017. However, no measurable heating was detected. Therefore, the possibility to extract mesospheric local gas temperatures from high-resolution sprite spectra makes sprites a natural thermal probe of (generally difficult to access) regions of the mesosphere located at different altitudes. However, there is also a lack of statistics in our gas temperature measurements. To probe the mesosphere temperature with more accuracy by means of fitting TLE spectra, we would need a larger amount of data.

It is also found that the altitude dependence of vibrational populations in multicolumn sprites is similar to the one previously reported within carrot sprites. However, the carrot sprite altitude-dependent VDF trend is not completely fulfilled when comparing the VDFs from single-column and multicolumn sprites recorded at various altitudes. No spectroscopic features of Meinel $\mathrm{N}_{2}^{+}$nor $777.4 \mathrm{~nm}(\mathrm{O}$ I) optical emissions were found in any of our recorded high-resolution spectra of sprites at the altitudes explored.

\section{References}

Adachi, T., Hiraki, Y., Yamamoto, K., Takahashi, Y., Fukunishi, H., Hsu, R.-R., .. Lee, L. C. (2008). Electric fields and electron energies in sprites and temporal evolutions of lightning charge moment. Journal of Physics D: Applied Physics, 41(23), 234010. https://doi.org/10.1088/ 0022-3727/41/23/234010

Berk, A., Anderson, G. P., Acharya, P. K., Bernstein, L. S., Muratov, L., \& Lee, J. (2005). MODTRAN 5: A reformulated atmospheric band model with auxiliary species and practical multiple scattering options: Update. In Proceedings of the Society of Photo-Optical Instrumentation Engineers (SPIE) Conference, Society of Photo-Optical Instrumentation Engineers (SPIE) Conference Series (Vol. 5806, pp. 662-667). Orlando, FL. 
Betz, H., Schmidt, K., Laroche, P., Blanchet, P., Oettinger, W. P., Defer, E., ... Konarski, J. (2008). LINET—An international lightning detection network in Europe. Atmospheric Research, 91, 543-573. https://doi.org/10.1016/j.atmosres.2008.06.012

Biloiu, C., Sun, X., Harvey, Z., \& Scime, E. (2007). An alternative method for gas temperature determination in nitrogen plasmas: Fits of the bands of the first positive system $\left(\mathrm{B}^{3} \Pi_{g} \rightarrow \mathrm{A}^{3} \Sigma_{u}\right)$. Journal of Applied Physics, 101, 073303.

Bucsela, E., Morrill, J., Heavner, M., Siefring, C., Berg, S., Hampton, D., ... Sentman, D. (2003). $N_{2}\left(B^{3} \Pi_{g}\right)$ and $N_{2}^{+}\left(A^{2} \Pi_{u}\right)$ vibrational distributions observed in sprites. Journal of Atmospheric and Solar-Terrestrial Physics, 65, 583-590. https://doi.org/10.1016/ S1364-6826(02)00316-4

Franz, R. C., Nemzek, R. J., \& Winckler, J. R. (1990). Television image of a large upward electrical discharge above a thunderstorm system. Science, 249, 48-51. https://doi.org/10.1126/science.249.4964.48

García-Comas, M., López-Puertas, M., Marshall, B. T., Wintersteiner, P. P., Funke, B., Bermejo-Pantaleón, D., ... Russell, J. M. (2008). Errors in Sounding of the Atmosphere using Broadband Emission Radiometry (SABER) kinetic temperature caused by non-local-thermodynamic-equilibrium model parameters. Journal of Geophysical Research, 113, D24106. https://doi.org/10.1029/ 2008JD010105

Gordillo-Vázquez, F. J. (2008). Air plasma kinetics under the influence of sprites. Journal of Physics D: Applied Physics, 41 (23), 234016. https://doi.org/10.1088/0022-3727/41/23/234016

Gordillo-Vázquez, F. J., Luque, A., \& Simek, M. (2011). Spectrum of sprite halos. Journal of Geophysical Research, 116, A09319. https://doi.org/10.1029/2011JA016652

Gordillo-Vázquez, F. J., Luque, A., \& Simek, M. (2012). Near infrared and ultraviolet spectra of TLEs. Journal of Geophysical Research, 117, A05329. https://doi.org/10.1029/2012JA017516

Hallinan, T. J., Stenbaek-Nielsen, H. C., \& Deehr, C. S. (1985). Enhanced aurora. Journal of Geophysical Research, 90, 8461-8475. https://doi.org/10.1029/JA090iA09p08461

Hampton, D. L., Heavner, M. J., Wescott, E. M., \& Sentman, D. D. (1996). Optical spectral characteristics of sprites. Geophysical Research Letters, 23, 89-92. https://doi.org/10.1029/95GL03587

Hauchecorne, A., Chanin, M. L., \& Keckhut, P. (1991). Climatology and trends of the middle atmospheric temperature (33-87 km) as seen by Rayleigh lidar over the south of France. Journal of Geophysical Research, 96(D8), 15,297-15,309.

Heavner, M. J., Morrill, J. S., Siefring, C., Sentman, D. D., Moudry, D. R., Wescott, E. M., \& Bucsela, E. J. (2010). Near-ultraviolet and blue spectral observations of sprites in the 320-460 nm region: $\mathrm{N}_{2}$ (2PG) emissions. Journal of Geophysical Research, 115, A00E44. https://doi.org/10.1029/2009JA014858

Herzberg, G. (1950). Molecular spectra and molecular structure I. Spectra of diatomic molecules. New York: Van Nostrand Reinhold Co.

Hiraki, Y. (2010). The phase transition theory of sprite halo. Journal of Geophysical Research, 115, A00E20. https://doi.org/10.1029/ 2009JA014384

Kanmae, T., Stenbaek-Nielsen, H. C., \& McHarg, M. G. (2007). Altitude resolved sprite spectra with 3 ms temporal resolution. Geophysical Research Letters, 34, L07810. https://doi.org/10.1029/2006GL028608

Kanmae, T., Stenbaek-Nielsen, H. C., McHarg, M. G., \& Haaland, R. K. (2010a). Observation of blue sprite spectra at 10,000 fps. Geophysical Research Letters, 37, L13808. https://doi.org/10.1029/2010GL043739

Kanmae, T., Stenbaek-Nielsen, H. C., McHarg, M. G., \& Haaland, R. K. (2010b). Observation of sprite streamer head's spectra at 10,000 fps. Journal of Geophysical Research, 115, A00E48. https://doi.org/10.1029/2009JA014546

Liu, N., \& Pasko, V. P. (2005). Molecular nitrogen LBH band system far-UV emissions of sprite streamers. Geophysical Research Letters, 32, L05104. https://doi.org/10.1029/2004GL022001

Lopez-Gonzalez, M., García-Comas, M., Rodríguez, E., López-Puertas, M., Shepherd, M. G., Shepherd, G. G., ... Wiens, R. H. (2007). Ground-based mesospheric temperatures at mid-latitude derived from $\mathrm{O}_{2}$ and $\mathrm{OH}$ airglow SATI data: Comparison with SABER measurements. Journal of Atmospheric and Solar-Terrestrial Physics, 69, 2379-2390.

Luque, A., \& Ebert, U. (2009). Emergence of sprite streamers from screening-ionization waves in the lower ionosphere. Nature Geoscience, 2(757-760). https://doi.org/10.1038/ngeo662

Luque, A., \& Ebert, U. (2010). Sprites in varying air density: Charge conservation, glowing negative trails and changing velocity. Geophysical Research Letters, 37, L06806. https://doi.org/10.1029/2009GL041982

Luque, A., \& Gordillo-Vázquez, F. J. (2011). Modeling and analysis of $\mathrm{N}_{2}\left(\mathrm{~B}^{3} \Pi_{g}\right)$ and $\mathrm{N}_{2}\left(\mathrm{C}^{3} \Pi_{u}\right)$ vibrational distributions in sprites. Journal of Geophysical Research, 116, A02306. https://doi.org/10.1029/2010JA015952

Mende, S. B., Rairden, R. L., Swenson, G. R., \& Lyons, W. A. (1995). Sprite spectra; N 1 PG band identification. Geophysical Research Letters, 22 , 2633-2636. https://doi.org/10.1029/95GL02827

Morrill, J. S., Bucsela, E. J., Pasko, V. P., Berg, S. L., Heavner, M. J., Moudry, D. R., ... Sentman, D. D. (1998). Time resolved N 2 triplet state vibrational populations and emissions associated with red sprites. Journal of Atmospheric and Solar-Terrestrial Physics, 60(7-9), 811-829. https://doi.org/10.1016/S1364-6826(98)00031-5

Parra-Rojas, F. C., Luque, A., \& Gordillo-Vázquez, F. J. (2013). Chemical and electrical impact of lightning on the Earth mesosphere: The case of sprite halos. Journal of Geophysical Research: Space Physics, 118, 1-25. https://doi.org/10.1002/jgra.50449

Parra-Rojas, F. C., Luque, A., \& Gordillo-Vázquez, F. J. (2015). Chemical and thermal impact of sprite streamers in the Earth mesosphere. Journal of Geophysical Research: Space Physics, 120, 8899-8933. https://doi.org/10.1002/2014JA020933

Parra-Rojas, F. C., Passas, M., Carrasco, E., Luque, A., Tanarro, I., Simek, M., \& Gordillo-Vázquez, F. J. (2013). Spectroscopic diagnosis of laboratory air plasmas as a benchmark for spectral diagnosis of TLEs. Journal of Geophysical Research: Space Physics, 118, 4649-4661. https://doi.org/10.1002/jgra.50433

Pasko, V. P., Inan, U. S., \& Bell, T. F. (1998). Spatial structure of sprites. Geophysical Research Letters, 25, 2123-2126. https://doi.org/10.1029/98GL01242

Passas, M., Madiedo, J. M., \& Gordillo-Vázquez, F. J. (2016). High resolution spectroscopy of an Orionid meteor from 700 to 800 nm. Icarus, 266, 134-141. https://doi.org/10.1016/j.icarus.2015.11.020

Passas, M., Sánchez, J., Luque, A., \& Gordillo-Vázquez, F. J. (2014). Transient upper atmospheric plasmas: Sprites and halos. IEEE Transactions on Plasma Science, 42, 2664-2665. https://doi.org/10.1109/TPS.2014.2329320

Passas, M., Sánchez, J., Sánchez-Blanco, E., Luque, A., \& Gordillo-Vázquez, F. J. (2016). GRASSP: A spectrograph for the study of transient luminous events. Applied Optics, 55(23), 6436-6442. https://doi.org/10.1364/AO.55.006436

Sentman, D. D., Stenbaek-Nielsen, H. C., McHarg, M. G., \& Morrill, J. S. (2008). Plasma chemistry of sprite streamers. Journal of Geophysical Research, 113, D11112. https://doi.org/10.1029/2007JD008941

São Sabbas, F. T., Sentman, D. D., Wescott, E. M., Pinto, O. Jr., Mendes, O. Jr., \& Taylor, M. J. (2003). Statistical analysis of space-time relationships between sprites and lightning. Journal of Atmospheric and Terrestrial Physics, 65, 525-535. https://doi.org/10.1016/ S1364-6826(02)00326-7 
Simek, M. (1994). On the use of the numerical simulation of the first positive system of $\mathrm{N}_{2}$ : III. Numerical thermometer on $\left(v^{\prime}, 0\right)$ bands, $v^{\prime}=0-3$ (Research Report IPPCZ-345) (pp. 1-31).

Simek, M., \& De Benedictis, S. (1995). On the use of the numerical simulation of the first positive system of $\mathrm{N}_{2}:$ :ll. Fast $T_{\text {rot }}$ estimation from the partially resolved $(3,0)$ band. Plasma Chemistry and Plasma Processing, 15, 451-463.

Stenbaek-Nielsen, H. C., Haaland, R., McHarg, M. G., Hensley, B. A., \& Kanmae, T. (2010). Sprite initiation altitude measured by triangulation. Journal of Geophysical Research, 115, A00E12. https://doi.org/10.1029/2009JA014543

Stenbaek-Nielsen, H. C., McHarg, M. G., Kanmae, T., \& Sentman, D. D. (2007). Observed emission rates in sprite streamer heads. Geophysical Research Letters, 34, L11105. https://doi.org/10.1029/2007GL029881

van der Velde, O. A., \& Montanyà, J. (2016). Statistics and variability of the altitude of elves. Geophysical Research Letters, 43, 5467-5474. https://doi.org/10.1002/2016GL068719

Williams, E. (2012). Resolution of the sprite polarity paradox: The role of halos. Radio Science, 47, RS2002. https://doi.org/ 10.1029/2011RS004794 